\title{
Caracterização Viscosimétrica de Nanocompósitos Híbridos PS/POSS
}

\author{
Otávio Bianchi \\ Universidade de Caxias do Sul, UCS \\ Laboratório de Polímeros Avançados, Instituto de Química, UFRGS \\ Gustavo B. Repenning, Raquel S. Mauler, Ricardo V. B. Oliveira \\ Laboratório de Polímeros Avançados, Instituto de Química, UFRGS \\ Leonardo B. Canto \\ Departamento de Engenharia de Materiais, UFSCar
}

\begin{abstract}
Resumo: Nanocompósitos híbridos de poliestireno (PS) e poliedros oligoméricos silsesquioxanos (POSS) com diferentes composições e graus de hibridização foram obtidos por processamento reativo no estado fundido utilizando-se peróxido de dicumila (DCP) como iniciador, na presença ou não de estireno como agente de transferência de radical. Os materiais foram caracterizados viscosimetricamente por cromatografia de permeação em gel (GPC) usando detecção tripla por espalhamento de luz, viscosimetria e índice de refração. As amostras PS/POSS processadas com estireno apresentaram maiores valores de massa molar ponderal média $\left(\mathrm{M}_{\mathrm{w}}\right)$ e menores valores de polidispersão $\left(\mathrm{M}_{\mathrm{w}} / \mathrm{M}_{\mathrm{n}}\right)$, devido ao maior grau de conversão da reação de hibridização do PS-POSS (28-40\%) e do menor grau de degradação (cisão) das cadeias do PS, quando comparadas com amostras PS/POSS processadas sem estireno nas quais o grau de conversão ficou em torno de 24-28\%. Para os sistemas PS e PS/POSS em solução com THF, os parâmetros da equação de Mark-Houwink-Sakurada (MHS), $\alpha \cong 0,7$ e $\log K \cong-3,5$ a $-3,9$ e os valores dos parâmetros de interação polímero-solvente, $\chi_{\mathrm{ij}} \cong 0,49$, não apresentaram diferenças significativas com relação aos tamanhos moleculares. Por outro lado, essas diferenças de tamanhos moleculares foram caracterizadas por uma função cumulativa da fração mássica de cadeias em função da distância média quadrática entre pontas de cadeia $\left(<r^{2}>_{0}{ }^{1 / 2}\right)$.
\end{abstract}

Palavras-chave: PS, POSS, nanocompósitos híbridos, viscosimetria.

\section{Viscometric Characterization of PS/POSS Hybrid Nanocomposites}

Abstract: Polystyrene (PS) and polyhedral oligomeric silsesquioxanes (POSS) hybrid nanocomposites with different compositions were obtained by reactive melt processing using dicumyl peroxide (DCP) as initiator in the presence or absence of styrene as radical transfer agent. The materials were characterized by viscosimetry by means of gel permeation chromatography (GPC) using triple-detector: light scattering, viscometer and refractive index. PS/POSS samples processed with styrene showed higher weight average molecular weights $\left(\mathrm{M}_{\mathrm{w}}\right)$ and lower polydispersity indexes $\left(\mathrm{M}_{\mathrm{w}} / \mathrm{M}_{\mathrm{n}}\right)$, as a result of higher PS-POSS conversion (28-40\%) and lower PS degradation, as compared to the PS/POSS samples processed without styrene in which the degree of conversion was lower (24-28\%). For the PS/POSS solutions in THF, the parameters of the Mark-Houwink-Sakurada equation, $\alpha \cong 0.7$ and $\log K \cong-3.5$ to -3.9 , and the values of polymer-solvent interaction parameter, $\chi_{\mathrm{ij}} \cong 0.49$, were not changed with respect to changes in molecular size. On the other hand, these changes were characterized by a cumulative function of the mass fraction of chains as a function of the root mean square end to end distance $\left(<r^{2}>_{0}^{1 / 2}\right)$.

Keywords: PS, POSS, hybrid nanocomposites, viscosimetry.

\section{Introdução}

A utilização de poliedros oligoméricos silsesquioxanos (POSS) em reações de copolimerização, enxertia ou mistura no estado fundido vem sendo explorada para a melhoria de propriedades mecânicas e térmicas de polímeros convencionaii ${ }^{[1-4]}$. As moléculas híbridas de POSS são formadas basicamente por estruturas com tamanho nanométrico com ligações silício e oxigênio, com fórmula: $-\left(\mathrm{SiO}_{1,5}\right)_{\mathrm{n}}-$.

A inserção de grupos orgânicos nas moléculas de POSS pode torná-lo solúvel em solventes orgânicos e compatível com polímeros ${ }^{[5]}$. A inserção de grupos funcionais nas moléculas de POSS possibilita reações de copolimerização e enxertia com polímeros. O processamento reativo no estado fundido é um método técnico e economicamente viável e ecologicamente interessante para estes propósitos. Pode ser realizado através de equipamentos de processamento convencionais, além de não necessitar da utilização de solvente como meio reacional ${ }^{[6-8]}$.
Zhou et al. ${ }^{[9]}$ estudaram a enxertia de POSS em polipropileno (PP) por processamento reativo no estado fundido utilizando peróxido de dicumila (DCP) como iniciador da reação. A reação de enxertia de POSS contendo grupo amina com poliestireno funcionalizado com anidrido maleico (PS-MAH) foi estudada por Monticelli et al. ${ }^{[10]}$. Os autores observaram a formação de uma morfologia de fases separadas, formada por matriz de PS com agregados de POSS e moléculas enxertadas PS-graft-POSS. O material apresentou boa estabilidade térmica e melhoria nas propriedades mecânicas comparado ao PS puro.

A conversão dessas reações pode ser quantificada por medidas viscosimétricas ou medidas espectroscópicas ${ }^{[11]}$. As modificações moleculares causadas por tais reações podem ser avaliadas por medidas de cromatografia de permeação em gel (GPC). O uso de detectores como viscosímetros on-line e espalhamento de luz 
tornou essa técnica atrativa para a determinação de massas molares absolutas e outras propriedades de polímeros em solução ${ }^{[12-14]}$.

Através de medidas viscosimétricas on-line em conjunto com GPC, Ouano ${ }^{[15]}$ obteve bons ajustes para a equação de Mark-Houwink-Sakurada (MHS). Os parâmetros $K$ e $\alpha$ obtidos dessa equação são influenciados pela magnitude da massa molar, polidispersão, ramificações, rigidez das moléculas e pela interação polímero-solvente ${ }^{[16,17]}$. As propriedades físico-químicas de polímeros em solução como tamanho de cadeia e distância entre finais de cadeia são fortemente dependentes da interação polímero-solvente, temperatura e concentração da solução ${ }^{[18]}$. Essas propriedades são comumente determinadas por medidas de viscosidade intrínseca ([ๆ]) em solução diluída em condição teta $(\theta)$, nas quais o novelo polimérico encontra-se no estado não perturbado $^{[19-21]}$.

Stockmayer e Fixman ${ }^{[22]}$ mostraram que algumas dimensões da cadeia polimérica no estado não perturbado podem ser estimadas através de medidas de viscosidade intrínseca de soluções fora das condições $\theta$. Esta aproximação pode ser feita quando os efeitos hidrodinâmicos são desprezíveis. Dessa maneira, é possível conhecer o coeficiente de expansão do novelo $\left(\alpha_{n}\right)$ e determinar o valor de $K_{0}$ que é uma constante na qual estão contidas informações sobre a distância entre pontas de cadeia em condições não perturbadas.

O objetivo deste estudo é caracterizar nanocompósitos híbridos de poliestireno (PS) e poliedro oligomérico silsesquioxano (POSS) obtidos por processamento reativo no estado fundido por meio de medidas viscosimétricas usando cromatografia de permeação em gel (GPC) com detecção tripla: por espalhamento de luz, viscosimetria e índice de refração. Os valores de massa molar ponderal média $\left(\mathrm{M}_{\mathrm{w}}\right)$, polidispersão $\left(\mathrm{M}_{\mathrm{n}} / \mathrm{M}_{\mathrm{w}}\right)$, os parâmetros da equação de Mark-Houwink-Sakurada e os valores das distâncias médias quadráticas entre pontas de cadeia $\left(\left\langle r^{2}\right\rangle_{0}^{1 / 2}\right)$ foram correlacionados com o grau de conversão da reação de hibridização do PS-POSS e com o nível de degradação do PS em função das condições de processamento reativo empregadas para os sistemas PS/POSS

\section{Experimental}

\section{Materiais}

Poliestireno atático foi adquirido da Sigma-Aldrich (441147). Apresenta massa molar ponderal média $\left(M_{w}\right)$ de 267.000 g.mol ${ }^{-1}$, polidispersão $\left(M_{w} / M_{n}\right)$ de 2 , conforme análises por cromatografia de permeação em gel (GPC). Metacrilato de 3-(heptafenil-POSS)-propila (Methacryl Phenyl POSS, MA 0734-T8), foi adquirido da Hybrid Plastics (USA) na forma de pó cristalino, com massa molar de $1.083 \mathrm{~g} \cdot \mathrm{mol}^{-1}$. O POSS possui um grupo metacrílico e sete fenilas ligadas aos átomos de silício, conforme ilustrado na Figura 1. Peróxido de dicumila (DCP) com pureza de $98 \%$ foi adquirido da Sigma-Aldrich. O monômero
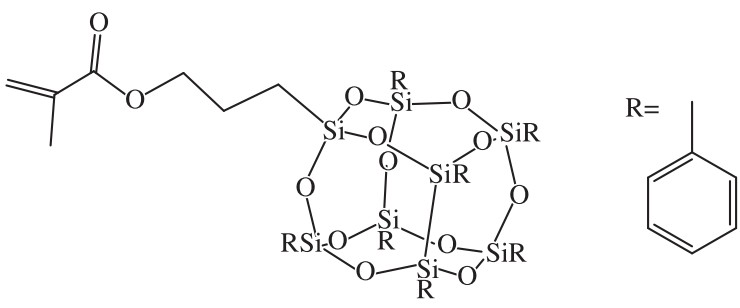

Figura 1. Estrutura do metacrilato de 3-(heptafenil-POSS)-propila. estireno com pureza de $99 \%$ foi adquirido da Sigma-Aldrich. Foi realizada a retirada de inibidor através de destilação a vácuo.

\section{Processamento reativo}

Os materiais foram preparados em câmara de mistura (Rheomix 600p) de reômetro de torque (Haake rheocord) com rotores do tipo roller, utilizando a velocidade dos rotores de $200 \mathrm{rpm}$, temperatura da câmara de $190{ }^{\circ} \mathrm{C}$, por um período 15 minutos. Utilizou-se $50 \mathrm{~g}$ de amostra por batelada.

As composições de PS/POSS processadas foram: 100/0, 99,5/0,5, 99/1, 98/2 e 95/5 (percentuais em massa). Empregou-se DCP como agente iniciador da reação de hibridização do PS-POSS na proporção DCP/POSS de 0,05 (g/g) em massa (razão molar DCP/POSS $=0,2$ ). Uma série de amostras foi preparada empregando-se monômero estireno como agente de transferência de radical da reação de hibridização do PS-POSS, na proporção de $2 \%$ em relação à massa total dos materiais.

\section{Cromatografia de permeação em gel (GPC)}

As análises de cromatografia de permeação em gel (GPC) foram realizadas em equipamento Viscotek modelo TDAmax ${ }^{\mathrm{TM}}$, equipado com um conjunto de 4 colunas dispostas em seqüência (Waters HR-4E, HR-4, HR-3 e HR-2), com fase estacionária constituída de microesferas de copolímeros de estireno-divinil benzeno (Ultrastyragel). A temperatura das colunas foi mantida em $45^{\circ} \mathrm{C}$. O solvente tetrahidrofurano (THF) foi usado como fase móvel a vazão de $1 \mathrm{~mL} / \mathrm{min}$. As soluções com concentração de $10 \mathrm{mg} / \mathrm{mL}$ foram injetadas com volume de $150 \mu \mathrm{L}$ e fluxo de solvente de $1 \mathrm{~mL} / \mathrm{min}$. Foram realizados experimentos em duplicata.

As análises foram realizadas empregando-se o sistema de detector triplo (Triple detector -TD), com detectores de espalhamento de luz, viscosímetro e índice de refração (IR). Utilizou-se o valor de 0,1850 para o incremento no índice de refração $(d n / d c)^{[23]}$. Através da utilização desse conjunto de detectores foi possível a determinação direta da massa molar por espalhamento de luz baseado nos princípios de espalhamento Rayleigh ${ }^{[13]}$, eliminado, assim, a necessidade de calibração da coluna com padrões conhecidos como os utilizados em análises de GPC convencionais. O uso do viscosímetro permitiu realizar medidas da viscosidade intrínseca ([ๆ]) online dos polímeros em solução. A variação de concentração foi mantida constante pelo uso do detector de índice de refração (IR), sendo necessária para determinação da distribuição de massas molares e viscosidade intrínseca ${ }^{[24]}$. O TD é capaz de realizar os mesmos procedimentos que a calibração universal faz, necessitando apenas de uma calibração para o offset de seus detectores. Através do TD foram obtidos valores de massa molecular absoluta em cada instante. Como resultado final foi obtido um amplo espectro de massas molares ${ }^{[24]}$.

O detector viscosimétrico utilizado nesse estudo é composto por quatro viscosímetros capilares de $0,25 \mathrm{~mm}$ ligados entre si, conforme esquematizado na Figura 2, na qual cada resistência $R i$ representa um viscosímetro.

Através da medida da diferença de pressão nos transdutores do meio da ponte DP e a diferença de pressão entre a entrada e saída IP é possível estabelecer a diferença de pressão total no circuito viscosimétrico. Considerando-se a lei de Poiseuille ${ }^{[25,26]}$ para escoamento laminar em tubos capilares é possível determinar a viscosidade da solução. Realizando-se uma série de simplificações e considerações que são descritas na literatura ${ }^{[13,24]}$, chega-se à uma expressão que relaciona as diferenças de pressão no viscosímetro com a viscosidade específica: 


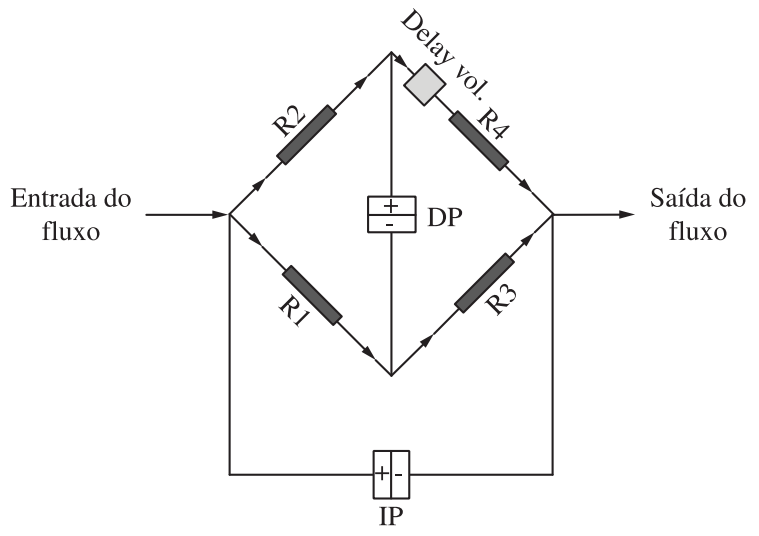

Figura 2. Esquema de circuito viscosímetro análogo a uma ponte de Wheatstone. Adaptado da referência ${ }^{[13]}$.

$$
\eta_{s p}=\frac{4 D P}{I P-2 D P}
$$

Como a cada instante o GPC está bombeando uma vazão de solvente conhecida o aumento ou redução da viscosidade da solução podem ser medidos ao longo do tempo de análise. A viscosidade reduzida, $\eta_{s p} / c$, na qual $c$ é a concentração do polímero na solução, é uma medida da capacidade específica do polímero de causar aumento na viscosidade relativa. $\mathrm{O}$ limite onde a diluição é infinita é conhecido como viscosidade intrínseca, [ $\eta]$, de acordo com a seguinte relação ${ }^{[13,14,25,27,28]}$ :

$$
[\eta]=\left(\frac{\eta_{s p}}{c}\right)_{c \rightarrow 0}
$$

Como as concentrações das soluções nas análises de GPC são baixas pode ser assumido que a solução é diluída. Dessa forma, é possível determinar a viscosidade intrínseca por meio dessas considerações.

A relação da viscosidade intrínseca com a massa molar de um polímero pode ser descrita através da relação empírica de Mark-Houwink-Sakurada ${ }^{[25,27]}$ :

$$
[\eta]=K \cdot M^{\alpha}
$$

$\mathrm{Na}$ Equação 3, $K$ e $\alpha$ são constantes empíricas que dependem da temperatura e da natureza da interação polímero/solvente ${ }^{[27]}$, e $M$ é a massa molar do polímero, nesse trabalho obtida através da utilização do TD. A constante $\alpha$ está relacionada com o tipo de conformação da cadeia polimérica em solução. Na condição não perturbada teta $(\theta)$, um polímero com cadeias lineares e flexíveis apresenta $\alpha$ igual a $0,5^{[14,29,30]}$. Quando há uma boa interação entre polímero e solvente, um polímero com cadeias lineares e flexíveis apresenta $\alpha$ entre 0,5 e 0,8 . Polímeros com cadeias rígidas apresentam valores de $\alpha$ maiores que $1^{[27]}$.

Os valores de $K$ e $\alpha$ podem ser obtidos a partir de um gráfico de $\log [\eta] \times \log [M]$. O coeficiente linear dessa curva é $\log K$ e o coeficiente angular é $\alpha$.

Em soluções com diluição tendendo ao infinito, as interações entre as cadeias poliméricas desaparecem e os efeitos relacionados ao volume excluído dos segmentos moleculares tornam-se desprezíveis. Em outras palavras, uma parte de uma molécula longa não pode ocupar o espaço referente à outra parte da mesma molécula $^{[20]}$. Nessas condições, é possível determinar a distância entre pontas da cadeia polimérica em solução.

Nesse estudo, as informações sobre as dimensões moleculares em condições não perturbadas e informações sobre as interações polímero-solvente foram derivadas da extrapolação proposta por Kurata-Stockmayer-Fixman (KSF) ${ }^{[20,21]}$ :

$$
[\eta] \cdot M^{-1 / 2}=K_{0}+0,51 \cdot B \cdot \Phi_{0} \cdot M^{1 / 2}
$$

Na Equação 4, $M$ é a massa molar do polímero, $K_{0}$ é uma constante que está relacionada com a distância entre pontas de cadeia em condições não perturbadas, $B$ é uma constante na qual estão contidas informações sobre o parâmetro de interação polímero-solvente $\left(\chi_{\mathrm{ij}}\right)$ e $\Phi_{0}$ é a constante universal de Flory $\left(2,1 \times 1023 \mathrm{~mol}^{-1}\right)^{[19]}$. O valor do parâmetro $B$ é maior quando a interação polímero solvente é favorecida por aumento de temperatura ou por grupos funcionais similares contidos no polímero e no solvente. Este comportamento pode ser semelhante ao encontrado para o segundo coeficiente virial determinado por experimentos de espalhamento de luz ${ }^{[21]}$, no qual sua magnitude é reflexo da interação polímero-solvente. $\mathrm{O}$ parâmetro $B$ pode ser definido por:

$$
B=\frac{2 v^{2} \cdot\left(1 / 2-\chi_{i j}\right)}{N_{A} \cdot V_{l}}
$$

$\mathrm{Na}$ Equação 5, v é o volume específico do polímero, $N_{\mathrm{A}}$ é número de Avogrado e $V_{1}$ é o volume molar do solvente, que para o THF é $81,095 \mathrm{~cm}^{3} \cdot \mathrm{mol}^{-1[31]}$.

Essas extrapolações semi-empíricas foram originalmente obtidas a partir de considerações teóricas do volume excluído, levando-se em consideração a viscosidade intrínseca e a massa molar do polímero ${ }^{[21]}$.

O método descrito acima foi utilizado para avaliar a magnitude da interação polímero-solvente em função das variáveis moleculares dos sistemas PS/POSS e também para obter informações sobre a distância entre pontas de cadeia em condições não perturbadas, a partir de medidas de GPC com detector viscosimétrico on-line.

Como o parâmetro $K_{0}$ pode ser definido em condições não perturbadas, é possível correlacionar esta constante com dimensões da macromolécula, como a distância média quadrática entre pontas de cadeia, através da seguinte relação ${ }^{[21]}$ :

$$
K_{0}=[\eta]_{0} \cdot M^{1 / 2}=\Phi_{0} \cdot\left(\frac{<r^{2}>}{M}\right)^{3 / 2}
$$

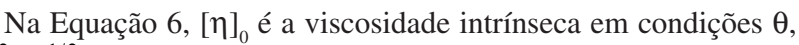
$<r^{2}>_{0}{ }^{1 / 2}$ é o valor quadrático médio da distância entre pontas de cadeia em condições não perturbadas ${ }^{[21,30]}$.

\section{Determinação do grau de conversão da reação do PS-POSS}

O grau de conversão da reação do PS-POSS nos nanocompósitos híbridos foi quantificado através de experimentos de $\mathrm{RMN}{ }^{1} \mathrm{H}$ e GPC, empregando-se uma curva de calibração produzida a partir de análises de padrões PS/POSS.

Os padrões PS/POSS foram preparados por dissolução de composições conhecidas de PS/POSS em THF (5\% em massa), seguida de homogeneização com auxílio de agitação mecânica e ultrasonificação por 6 horas. Em seguida, foram preparados filmes por evaporação do solvente. Os filmes foram secos em estufa a vácuo a $40{ }^{\circ} \mathrm{C}$ por 48 horas para remoção total do solvente.

Os espectros de $\mathrm{RMN}{ }^{1} \mathrm{H}$ foram obtidos em equipamento Varian Inova-300 . Cerca de $20 \mathrm{mg}$ de amostra foi dissolvida em $1 \mathrm{~mL}$ de $\mathrm{CDCl}_{3}$. O experimento foi conduzido a $22{ }^{\circ} \mathrm{C}$ utilizando-se como referência interna o tetrametilsilano (TMS). Nessas análises utilizou-se um pulso de $6,85 \mu$ s correspondendo a um ângulo de $45^{\circ}$, com um tempo de aquisição de 2,049 segundos, tempo de relaxação de 1 segundos e largura espectral de 4799,3 Hz. Foram efetuadas 128 varreduras em 6 minutos e 37 segundos. As medidas de $\mathrm{RMN}{ }^{1} \mathrm{H}$ permitiram quantificar o total de POSS presente nos sistemas 
PS/POSS, por meio do sinal do próton de éster $\left(-\mathrm{O}-\mathrm{CH}_{2}\right)$ do POSS com deslocamento em 4,2 ppm $^{[32,33]}$, conforme mostrado na Figura 3 a. A determinação quantitativa foi realizada levando em consideração os sinais dos deslocamentos químicos dos prótons aromáticos do $\operatorname{PS}^{[34]}$ e do POSS na região de 6,2 a 8 ppm. O procedimento de quantificação via $\mathrm{RMN}{ }^{1} \mathrm{H}$ utilizado nesse trabalho foi semelhante ao empregado por Zhang et al. ${ }^{[35]}$ para caracterizar os produtos da síntese de PS com diferentes tipos de POSS.

As análises de GPC foram realizadas conforme metodologia descrita anteriormente. Essas análises foram empregadas para medir a quantidade não reagida de POSS presentes nos sistemas PS/POSS. Essa determinação foi realizada através da integração da área relativa ao sinal do detector de índice de refração na região de aproximadamente 36 minutos, conforme mostrado na Figura $3 b$.

A curva de calibração foi construída baseada nas quantidades de POSS determinadas nos padrões pelas duas técnicas, conforme mostrado na Figura 3c. Dessa curva de calibração, obteve-se a relação matemática $\operatorname{POSS}_{R M N}(\%)=0,9509 \cdot \operatorname{POSS}_{G P C}(\%)$, através da qual foi possível relacionar a quantidade de POSS separada por GPC com a quantidade total de POSS determinada via RMN ${ }^{1} \mathrm{H}$ das amostras obtidas por processamento no estado fundido.

A conversão da reação de hibridização do PS-POSS nos nanocompósitos híbridos foi obtida através da seguinte Equação 7:

$$
\alpha_{P S-P O S S}(\%)=\frac{\left(P O S S_{R M N} / 0,9509\right)-P O S S_{G P C}}{\left(P O S S_{R M N} / 0,9509\right)} .100
$$

Na Equação 7, $\alpha_{\text {PS-Poss }}$ é o percentual de conversão da reação do PS-POSS nos nanocompósitos híbridos, $P O S S_{\mathrm{RMN}}$ é a quantidade percentual de POSS total presente na amostra determinada via RMN ${ }^{1} \mathrm{H}$ e $P O S S_{\mathrm{GPC}}$ é quantidade percentual de POSS não ligada ao $\mathrm{PS}$, que foi determinada por GPC.

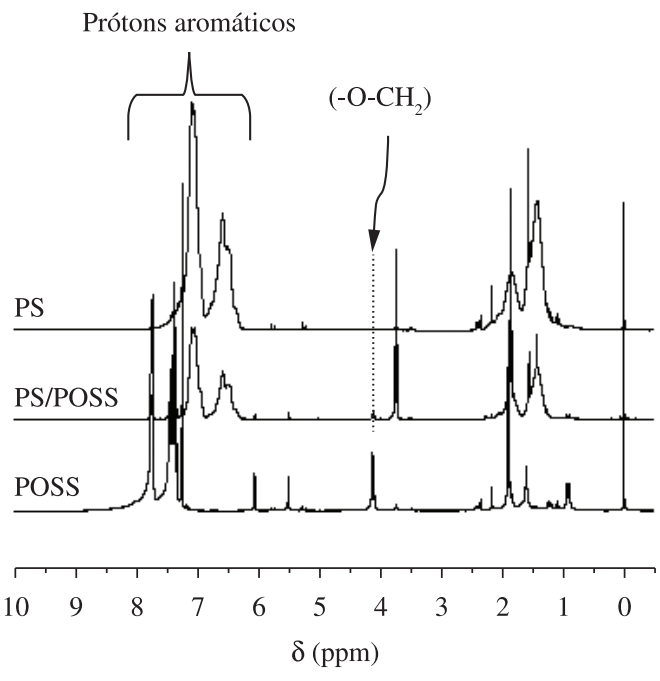

(a)

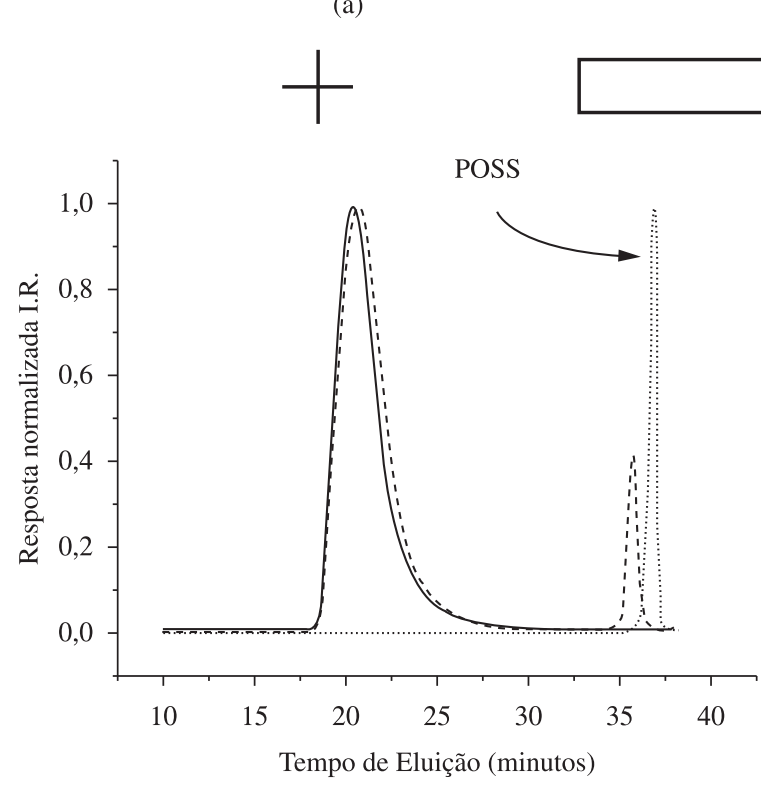

(b)

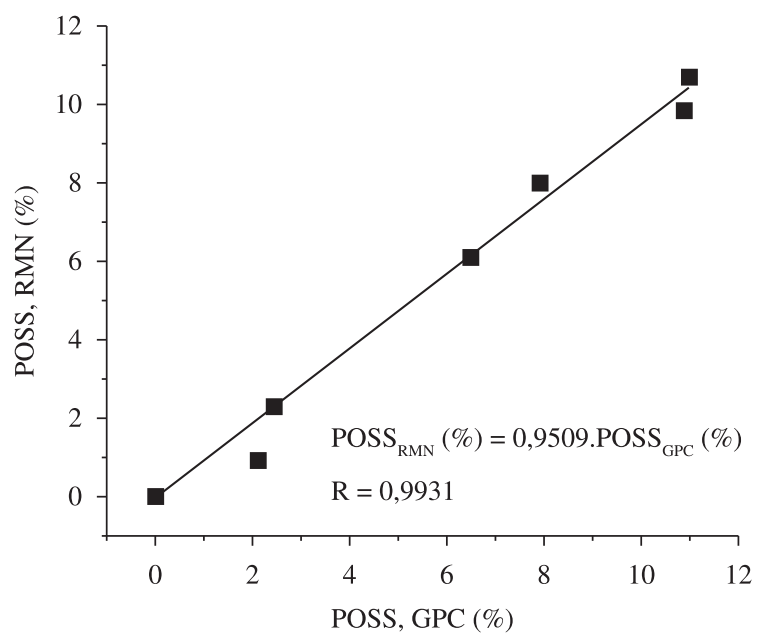

(c)

Figura 3. Método empregado para quantificação do grau de conversão de enxertia PS-graft-POSS nos nanocompósitos híbridos PS/POSS. a) espectro de RMN ${ }^{1} \mathrm{H}$; b) cromatograma de GPC; c) curva de calibração. 


\section{Resultados e Discussões}

A Tabela 1 apresenta os valores de massa molar ponderal média $\left(M_{w}\right)$ e de polidispersão $\left(M_{w} / M_{n}\right)$ obtidos por GPC, percentual total de POSS determinado por RMN ${ }^{1} \mathrm{H}$ (POSSRMN), percentual de POSS não reagido determinado por GPC $\left(P O S S_{\mathrm{GPC}}\right)$, e percentual de conversão da reação do PS-POSS ( $\left.\alpha_{\text {PS-Poss }}\right)$ nos nanocompósitos híbridos, obtido segundo a Equação 7.

As amostras PS/POSS processadas sem estireno apresentaram diminuição de $M_{w}$ e aumento da polidispersão com relação ao PS, o que é atribuído à cisão $\beta$ das cadeias de PS, que ocorre simultaneamente à reação de hibridização do PS-POSS, conforme o mecanismo proposto na Figura 4. Cabe lembrar que o PS empregado é um polímero não estabilizado e que o processamento utilizado emprega peróxido (DCP), fatos que contribuem para a degradação do PS, além da própria degradação termo-mecânica resultante do processamento. Para as amostras PS/POSS processadas sem estireno, o grau de conversão de PS-POSS apresentou valores da ordem de 24 a $28 \%$, com tendência de valores maiores para os sistemas PS/POSS contendo maiores teores de POSS.

As amostras PS/POSS processadas com estireno, de forma diferente, apresentaram aumento de $M_{w}$ e diminuição da polidispersão com relação ao PS. Esta classe de amostras apresentou maiores valores de conversão (28-40\%) com relação às amostras processadas sem estireno. Esse comportamento pode ser explicado devido ao fato de que o estireno age como agente de transferência de radical (Figura 4), aumentando o grau de enxertia e minimizando a degradação das cadeias de PS. Por isso, estas reações são denominadas reações de enxertia assistidas ou auxiliadas por estireno. Cabe ressaltar que o estireno adicionado pode também homopolimerizar, causando alteração na massa molar do sistema.

A adição de agente de transferência de radical é uma alternativa para o aumento do grau de conversão em sistemas poliméricos onde se pretende adicionar macromonômeros como o POSS, que possuem grande impedimento estérico. Um exemplo clássico de reação de enxertia na qual o estireno é adicionado para aumentar do grau de conversão é a reação de formação do polipropileno enxertado com anidrido maleico (PP-graft-MAH) ${ }^{[11,36]}$.

Zhou et al. ${ }^{[9]}$ obtiveram valores de conversão da reação enxertia de PP com POSS acima de 50\%, porém, utilizando-se de um POSS contendo oito grupos vinilas.

Na Figura 4 é apresentado um esquema simplificado das reações de enxertia e de degradação envolvidas no processamento reativo dos sistemas PS/POSS.

$\mathrm{Na}$ etapa (a) há formação de dois radicais livres por molécula de iniciador ${ }^{[37-39]}$. Esses radicais abstraem um átomo de hidrogênio da cadeia de PS dando origem ao macro-radical estirila (b) ${ }^{[40]}$. Os macro-radicais A podem reagir com o POSS (etapa c) levando a inserção deste na cadeia polimérica, ou com estireno levando a inserção de uma unidade monomérica na cadeia, originando o macroradical C. No entanto, também podem envolver a transferência de hidrogênio (d) e reações de cisão $\beta$ (e). Passaglia et al. ${ }^{[8]}$ obtiveram evidência de que a reação de cisão $\beta$ é favorecida para razões molares de monômero e iniciador (DCP) inferiores a 20. Neste caso (e) a degradação origina macro-radicais B e macromoléculas com terminais com dupla ligação. Além disso, o macro-radical B pode reagir com o POSS (f) ou estireno resultando em inserção nos terminais de cadeia.

A adição de monômero estireno no processamento do PS puro resulta no aparecimento de maior concentração de macro-radicais, que por sua vez acaba contribuindo para maximização das reações de cisão $\beta$. Por esse motivo, há redução de massa molar do PS na presença de estireno, conforme os valores de $\mathrm{M}_{\mathrm{w}}$ da Tabela 1. Entretanto, a adição de monômero estireno ao PS na presença do POSS, que possui um grande impedimento estérico, resulta na reação de enxertia com conseqüente aumento de massa molar. Este tipo de efeito de transferência de radical é observado na literatura na modificação do polipropileno (PP) com anidrido maleico (MAH) na presença de estireno ${ }^{[41,42]}$.

As curvas de viscosidade intrínseca em função da massa molar para amostras PS/POSS processadas sem e com estireno são apresentados nas Figuras 5a, b, respectivamente. As curvas apresentam duas regiões com inclinações distintas, com ponto de mudança em torno de 35.000 g. $\mathrm{mol}^{-1}$. Para todas as amostras, as curvas apresentam inclinações semelhantes para as frações de cadeias com massas molares acima de 35.000 g. $\mathrm{mol}^{-1}$, independentemente da quantidade de POSS ou do uso do estireno no processamento reativo. É importante ressaltar que a relação de Mark-Houwink-Sakurada (MHS) apresenta algumas limitações no seu uso em polímeros de baixa massa molar ( $\left.\mathrm{M} \leq 20.000 \mathrm{~g} \cdot \mathrm{mol}^{-1}\right)$. A relação não pode ser aplicada visto que as moléculas com baixa massa molar não possuem comportamento como cadeias gaussianas, mas sim como worn-like ${ }^{[43,44]}$. Outro fator que cabe comentar é que variações de concentração da solução alteram o coeficiente de fricção do solvente resultando em mudanças nos valores de [ $]$, fato que causa um grande impacto nas medidas de cadeias de baixa massa molar ${ }^{[45,46]}$. Esses comportamentos são evidenciados nos desvios observados nas curvas de viscosidade intrínseca para massas molares abaixo de $35.000 \mathrm{~g} \cdot \mathrm{mol}^{-1}$.

A resolução da equação de Mark-Houwink-Sakurada (Equação 3) foi realizada por regressão não-linear utilizando-se o algoritmo de Levenberg-Marquardt ${ }^{[47,48]}$. Os valores das constantes de ajustes da equação de Mark-Houwink-Sakurada ( $K$ e $\alpha$ ) para as

Tabela 1. Massas molares e grau de conversão nos nanocompósitos híbridos PS/POSS.

\begin{tabular}{|c|c|c|c|c|c|c|}
\hline PS/POSS & & $\begin{array}{c}* \mathbf{M}_{\mathrm{w}} \\
\left(\mathrm{g} \cdot \mathrm{mol}^{-1}\right)\end{array}$ & $\begin{array}{c}\text { *Polidispersão } \\
\left(\mathbf{M}_{w} / \mathbf{M}_{\mathrm{n}}\right)\end{array}$ & $\begin{array}{c}\text { POSS }_{\mathrm{RMN}} \\
(\mathrm{mol} \%)\end{array}$ & $\begin{array}{c}{ }^{* \mathrm{POSS}_{\mathrm{GPC}}} \\
(\% \text { em massa })\end{array}$ & $\begin{array}{c}\alpha_{\text {PS-POSS }} \\
(\mathbf{( \% )}\end{array}$ \\
\hline $100 / 0$ & \multirow{4}{*}{$\begin{array}{c}\text { Sem } \\
\text { estireno }\end{array}$} & 212,040 & 1,98 & - & - & - \\
\hline $99 / 1$ & & 205,760 & 2,01 & 0,75 & 0,60 & 23,9 \\
\hline $98 / 2$ & & 204,280 & 2,00 & 1,67 & 1,30 & 25,9 \\
\hline $95 / 5$ & & 200,830 & 2,17 & 4,34 & 3,30 & 27,6 \\
\hline $100 / 0$ & \multirow{5}{*}{$\begin{array}{l}2 \% \text { de } \\
\text { estireno }\end{array}$} & 195,209 & 2,36 & - & - & - \\
\hline $99,5 / 0,5$ & & 218,544 & 1,85 & - & 0,35 & - \\
\hline $99 / 1$ & & 221,076 & 1,88 & 0,86 & 0,65 & 28,1 \\
\hline $98 / 2$ & & 236,347 & 1,88 & 1,82 & 1,30 & 32,1 \\
\hline $95 / 5$ & & 240,783 & 1,82 & 4,55 & 2,85 & 40,4 \\
\hline
\end{tabular}

*Medidas de GPC com variação menor que 5\% em relação a média. 


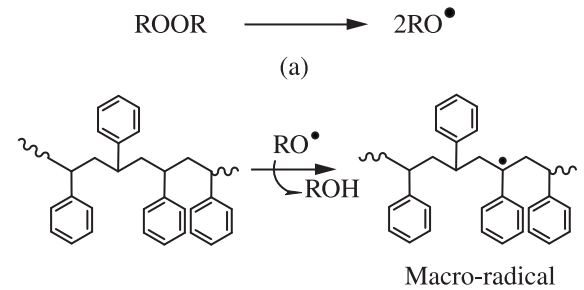

(b)

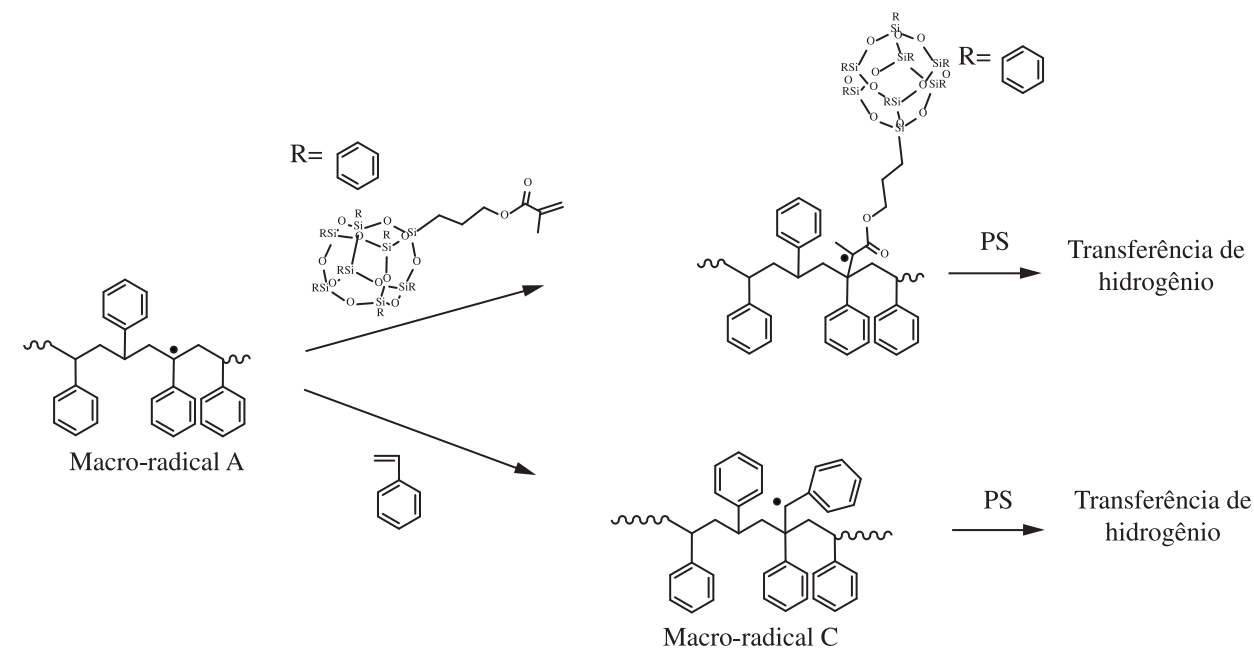

(c)<smiles>CC(CCc1ccccc1)c1ccccc1CCC1CCC(C)c2cccc3cccc1c23</smiles>

Macro-radical A<smiles>CC1CC(CCCCc2ccccc2)c2cccc3cccc1c23</smiles><smiles>CC(CCCc1ccccc1)CCc1cccc2ccccc12</smiles>

PS<smiles>CCC(CCCCc1ccccc1)Cc1cccc2cccc(C)c12</smiles>

Macro-radical A

(d)<smiles>CCC(CC(=O)c1ccccc1)c1cccc2cccc(CC(C)C)c12</smiles>

Macro-radical A

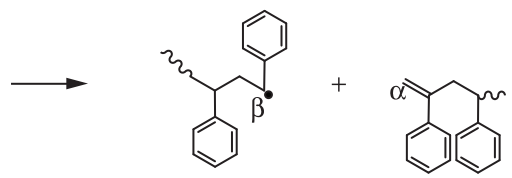

Macro-radical B

(e)

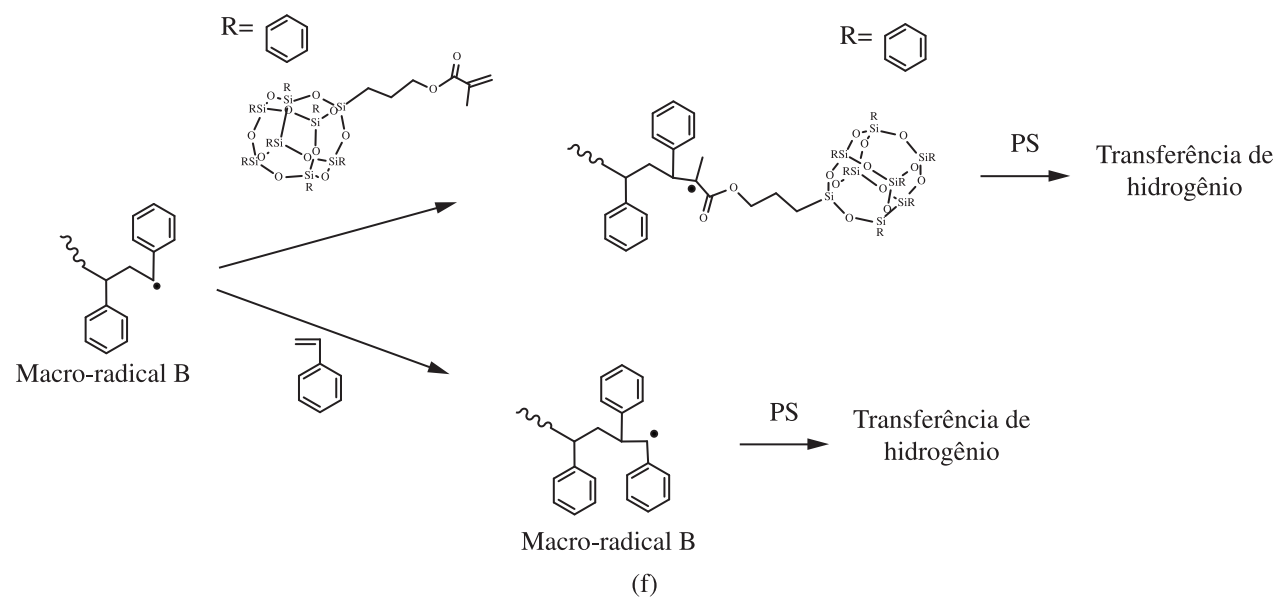

(f)

Figura 4. Reações de hibridização do PS-POSS e de degradação do PS envolvidas no processamento reativo dos sistemas PS/POSS. 
frações PS/POSS com massas molares acima de 35.000 g.mol ${ }^{-1}$ são apresentados na Tabela 2 .

Os valores de $\alpha$ obtidos para as frações com massas molares acima de 35.000 g. $\mathrm{mol}^{-1}$ são da ordem de 0,7 , os quais são próximos dos valores apresentados na literatura para o poliestireno em $\mathrm{THF}^{[49]}$. Os valores de $\log K$ ficaram entre $-3,5$ e $-3,9$, não apresentando diferenças significativas entre eles. Kurata et al..$^{[14]}$ obtiveram para o poliestireno atático em solução com THF a $25{ }^{\circ} \mathrm{C}$ valores de $\alpha$ entre 0,70 e 0,725 e valores de $l o g$ $K$ entre $-1,85$ e $-1,95$. Weber et al. ${ }^{[50]}$ em seu estudo de reações de enxertia de poliestireno em fulereno (C60) obtiveram valores de $\alpha$ igual a 0,71 e de $\log K$ igual a $-2,87$.

Os parâmetros termodinâmicos $B$ e $K_{0}$ estimados segundo a aplicação da Equação 4 são apresentados na Tabela 3. Os valores dos parâmetros $B$ e $K_{0}$ apresentaram pequena variação, não significativa, nos sistemas PS/POSS estudados.

Como os valores de $B$ (Tabela 3 ) estão na mesma grandeza decimal, os valores dos parâmetros de interação polímeros-solvente $\left(\chi_{\mathrm{ij}}\right)$ dos sistemas PS/POSS, obtidos segundo a Equação 5, são da ordem de 0,49 . Esse valor é semelhante ao apresentado na literatura para o sistema PS/THF ${ }^{[51]}$. Valores de $\chi_{\mathrm{ij}}<0,5$ refletem uma boa interação polímero-solvente ${ }^{[20]}$.

A técnica de GPC utilizada nesse trabalho permitiu medir a viscosidade intrínseca para cada fração de macromoléculas. Dessa

Tabela 2. Constantes de ajustes da equação de Mark-Houwink-Sakurada.

\begin{tabular}{ccccc}
\hline PS/POSS & & $\log K$ & $\alpha$ & $\mathbf{r}^{*}$ \\
\hline $100 / 0$ & & $-3,86 \pm 0,11$ & $0,70 \pm 0,02$ & 0,9954 \\
$99,5 / 0,5$ & & $-3,75 \pm 0,09$ & $0,69 \pm 0,05$ & 0,9960 \\
$99 / 1$ & Sem & $-3,88 \pm 0,12$ & $0,71 \pm 0,02$ & 0,9978 \\
$98 / 2$ & estireno & $-3,88 \pm 0,11$ & $0,71 \pm 0,03$ & 0,9947 \\
$95 / 5$ & & $-3,76 \pm 0,09$ & $0,69 \pm 0,04$ & 0,9988 \\
\hline $100 / 0$ & & $-3,67 \pm 0,01$ & $0,68 \pm 0,01$ & 0,9981 \\
$99,5 / 0,5$ & \multirow{2}{*}{ de } & $-3,52 \pm 0,01$ & $0,65 \pm 0,01$ & 0,9987 \\
$99 / 1$ & estireno & $-3,68 \pm 0,01$ & $0,68 \pm 0,01$ & 0,9996 \\
$98 / 2$ & & $-3,88 \pm 0,01$ & $0,71 \pm 0,01$ & 0,9992 \\
$95 / 5$ & & $-3,92 \pm 0,02$ & $0,72 \pm 0,01$ & 0,9966 \\
\hline$*$ Os ajustes obtiveram coeficiente de correlac̃ $(\mathrm{r})$ acima de $0,99$.
\end{tabular}

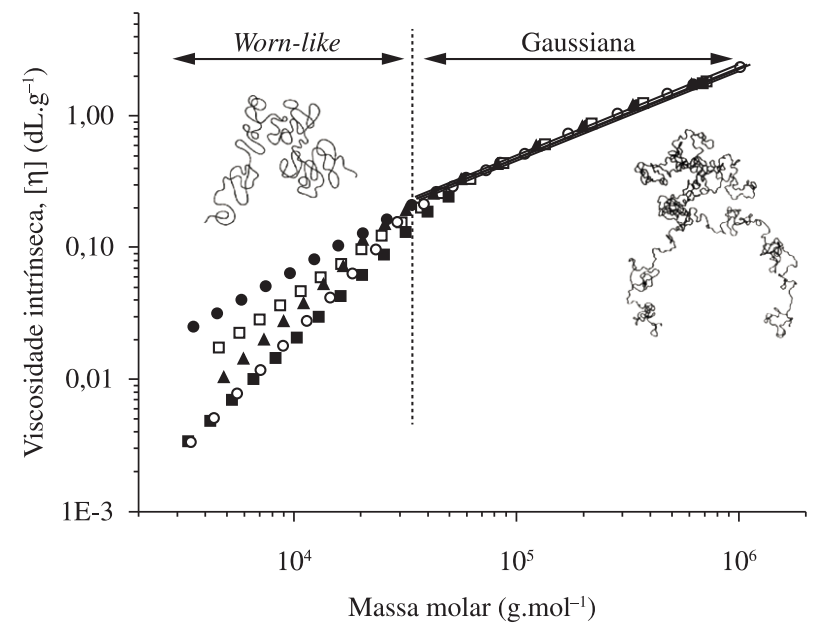

(a) maneira, a partir da Equação 6 e dos dados da Tabela 3 foi possível exprimir os valores das distâncias médias quadráticas entre pontas de cadeia $\left\langle r^{2}\right\rangle_{0}{ }^{1 / 2}$ através de curvas de distribuição, e não como uma simples média aritmética. A Figura 6 apresenta curvas de distribuição de $\left\langle r^{2}\right\rangle_{0}{ }^{1 / 2}$ de amostras de PS e PS/POSS 95/5 processadas sem estireno (a) e com $2 \%$ de estireno (b).

As curvas das amostras processadas sem estireno apresentaram uma maior concentração de cadeias expressa em fração mássica (FM) com valor de $\left\langle r^{2}\right\rangle_{0}^{1 / 2}$ em aproximadamente $8,3 \mathrm{~nm}$. Porém, a curva da amostra PS/POSS 95/5 apresentou uma fração maior de moléculas com menores valores de $\left\langle r^{2}>_{0}^{1 / 2}\right.$ e, conseqüentemente, uma fração menor de moléculas com maiores valores de $\left\langle r^{2}>_{0}^{1 / 2}\right.$. Este resultado está relacionado com a redução de massa molar (Tabela 1) observada para o sistema PS/POSS processado sem estireno, decorrente do processo de degradação do PS.

Para a classe de amostras processadas com estireno, observase deslocamento da curva da amostra PS/POSS 95/5 para valores maiores de $\left\langle r^{2}\right\rangle_{0}^{1 / 2}$ com relação à amostra PS. Observa-se também que o valor de $\left\langle r^{2}\right\rangle_{0}^{1 / 2}$ onde $F M / d \log M$ é máxima passou de $8,3 \mathrm{~nm}$ para o PS para 9,2 $\mathrm{nm}$ para a amostra PS/POSS 95/5. Portanto, fica evidente para esta classe de amostras que houve

Tabela 3. Parâmetros termodinâmicos estimados segundo o método $\mathrm{KSF}^{[21]}$.

\begin{tabular}{cccc}
\hline PS/POSS & & $\begin{array}{c}B \times \mathbf{1 0 3 0} \\
\left(\mathbf{m o l}^{\mathbf{2}} \mathbf{.} \mathbf{g}^{-2} \cdot \mathbf{d L}\right)\end{array}$ & $\begin{array}{c}K_{\mathbf{0}} \times \mathbf{1 0 4} \\
\left(\mathbf{m o l}^{\mathbf{1} / 2} \cdot \mathbf{g}^{\mathbf{2}} \mathbf{. d} \mathbf{d}\right)\end{array}$ \\
\hline $100 / 0$ & & 5,52 & 16,8 \\
$99,5 / 0,5$ & Sem & 6,49 & 17,1 \\
$99 / 1$ & estireno & 7,92 & 15,1 \\
$98 / 2$ & & 5,32 & 17,1 \\
$95 / 5$ & & 7,83 & 15,1 \\
\hline $100 / 0$ & & 7,44 & 17,7 \\
$99,5 / 0,5$ & & 4,72 & 20,4 \\
$99 / 1$ & $2 \%$ de & 7,03 & 19,7 \\
$98 / 2$ & estireno & 8,17 & 17,3 \\
$95 / 5$ & & 10,79 & 17,1 \\
\hline
\end{tabular}

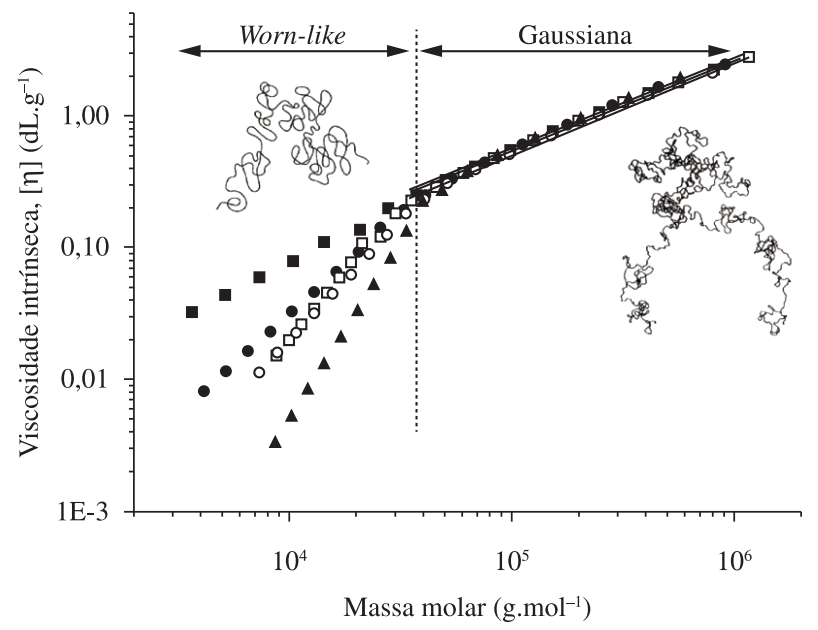

(b)

PS $\square$ PS/POSS 99,5/0,5 • PS/POSS 99/1 O PS/POSS 98/2 A PS/POSS 95/5 — Mark-Houwink-Sakurada

Figura 5. Viscosidade intrínseca em função da massa molar de amostras PS/POSS processadas sem estireno (a) e com $2 \%$ de estireno (b). 


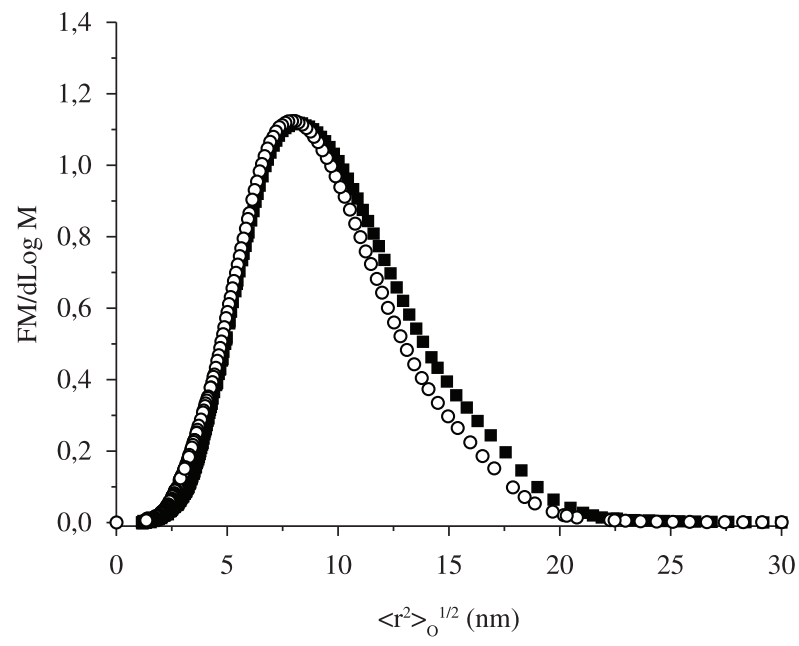

(a)

a PS/POSS 100/0 O PS/POSS 95/5

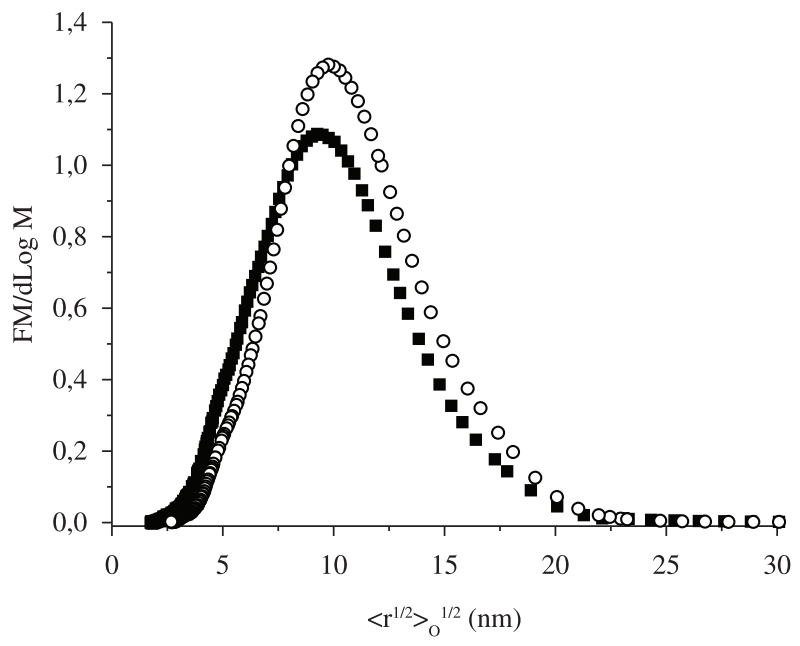

(b)

- PS O PS/POSS 95/5

Figura 6. Distribuição dos valores quadráticos médios da distância entre pontas de cadeia de amostras PS e PS/POSS 95/5 processadas sem estireno (a) e com $2 \%$ de estireno (b)

aumento na fração de moléculas com maior distância entre pontas de cadeia. Este fato está relacionado ao aumento de massa molar (Tabela 1) que foi observado para os sistemas processados com estireno causado pela maior conversão de hibridização do PS-POSS e devido ao menor grau de degradação do PS, quando comparado aos sistemas processados sem estireno.

Através de simulações computacionais utilizando modelo com aproximações grosseiras (Coarse-graining model) Spyriouni et al. ${ }^{[52]}$ mostraram para um PS linear com massa molar de aproximadamente $105.000 \mathrm{~g} . \mathrm{mol}^{-1}$ que a distância média quadrática entre pontas de cadeia é de $18 \mathrm{~nm}$ a $500 \mathrm{~K}$. Terao et al. ${ }^{[53]}$ obtiveram valores de $\left(<r^{2}>_{0}{ }^{1 / 2}\right)$ entre 2,4 e $3,8 \mathrm{~nm}$ para poliestirenos em ciclohexano a $34,5^{\circ} \mathrm{C}$ com massas molares entre 1.650 e 3.560 g.mol ${ }^{-1}$. Entretanto, cabe lembrar que os valores das distâncias médias quadráticas entre pontas de cadeia são influenciados pelo parâmetro de interação polímero-solvente e temperatura.

Alterações de tamanhos moleculares dos sistemas PS/POSS em termos de cisão de cadeias e reações de hidridização ocasionadas pelo processamento reativo não são evidentes nas curvas de viscosidade intrínseca em função da massa molar (Figura 5). Por outro lado, quando utilizada uma função cumulativa que leva em consideração a fração mássica de cadeias em função da distância entre pontas de cadeia, esses efeitos tornam-se mais evidentes (Figura 6).

\section{Conclusões}

Alterações de tamanhos moleculares em nanocompósitos de poliestireno (PS) e poliedros oligoméricos silsesquioxanos (POSS) preparados por processamento reativo no estado fundido com diferentes composições e graus de hibridização foram caracterizadas viscosimetricamente por cromatografia de permeação em gel (GPC) com sistema de detecção triplo, com detectores de espalhamento de luz, viscosímetro e índice de refração. As amostras PS/POSS processadas com DCP e com estireno apresentaram maiores valores de massa molar ponderal média $\left(\mathrm{M}_{\mathrm{w}}\right)$ e menores valores de polidispersão $\left(\mathrm{M}_{\mathrm{w}} / \mathrm{M}_{\mathrm{n}}\right)$, devido ao maior grau de conversão (28-40\%) da reação do PS-POSS e do menor grau de degradação (cisão) das cadeias do PS, quando comparadas com amostras PS/POSS processadas com DCP mas sem estireno nas quais o grau de conversão ficou em torno de 24-28\%. Esses dados evidenciam o efeito do monômero estireno como agente de transferência de radical no processo de hidridização do PS-POSS, aumentando o grau de conversão e minimizando a degradação do PS durante o processamento reativo. Para os sistemas PS e PS/POSS em solução com THF, os parâmetros da equação de Mark-Houwink-Sakurada $(\alpha$ e $K)$ e os valores dos parâmetros de interação polímero-solvente $\left(\chi_{\mathrm{ij}}\right)$ não apresentaram sensibilidade com relação às alterações de tamanhos moleculares decorrentes das diferenças de condições de processamento adotadas. Por outro lado, alterações de tamanhos moleculares em função das condições de processamento reativo foram caracterizadas por uma função cumulativa da fração mássica de cadeias em função da distância média quadrática entre pontas de cadeia $\left(<r^{2}>_{0}^{1 / 2}\right)$.

\section{Agradecimentos}

Ao Conselho Nacional de Desenvolvimento Científico e Tecnológico (CNPq) pela concessão da bolsa de doutorado a Otávio Bianchi.

\section{Referências Bibliográficas}

1. Baldi, F.; Bignotti, F.; Fina, A.; Tabuani, D. \& Ricco, T. - J. Appl. Polym. Sci., 105, p.935 (2007). http://dx.doi.org/10.1002/app.26142

2. Carniato, F.; Fina, A.; Tabuani, D. \& Boccaleri, E. - Nanotechnology, 19, p.1 (2008).

3. Joshi, M.; Butola, B. S.; Simon, G. \& Kukaleva, N. - Macromolecules, 39, p.1839 (2006). http://dx.doi.org/10.1021/ma051357w

4. Misra, R.; Alidedeoglu, A. H.; Jarrett, W. L. \& Morgan, S. E. - Polymer, 50, p.2906 (2009). http://dx.doi.org/10.1016/j.polymer.2009.03.057

5. Pielichowski, K.; Njuguna, J.; Janowski, B. \& Pielichowski, J. - "Polyhedral Oligomeric Silsesquioxanes (POSS) - containing nanohybrid polymers", in: Supramolecular Polymers Polymeric Betains Oligomers, Springer-Verlag Berlin, Berlin (2006).

6. Janssen, L. P. B. - "Reactive Extrusion Systems", Marcel Dekker, Inc. New York (2004). http://dx.doi.org/10.1201/9780203014172

7. Xanthos, M. - "Reactive Extrusion: Principles and Practice", Hanser Publishers, Munich (1992). 
8. Passaglia, E.; Coiai, S.; Ricci, L. \& Ciardelli, F. - Macromol. Symp., 218 (2004).

9. Zhou, Z.; Cui, L.; Zhang, Y.; Zhang, Y. \& Yin, N. - Eur. Polym. J., 44, p.3057 (2008). http://dx.doi.org/10.1016/j.eurpolymj.2008.05.036

10. Monticelli, O.; Fina, A.; Ullah, A. \& Waghmare, P. - Macromolecules, 42, p.6614 (2009). http://dx.doi.org/10.1021/ma900969b

11. Passaglia, E.; Coiai, S. \& Augier, S. - Prog. Polym. Sci., 34, p.911 (2009). http://dx.doi.org/10.1016/j.progpolymsci.2009.04.008

12. Greene, S. V. - "SEC with On-Line Triple Detection: Light Scattering,Viscometry, and Refractive Index", in: Encyclopedia of Chromatography, J. Cazes (ed.), Marcel Dekker, Inc., New York (2002).

13. Jackson, C. \& Barth, H. G. - "Molecular Weight Sensitive Detectors for Size Exclusion Chromatography", in: Handbook of Size Exclusion Chromatography and Related Techniques Second Edition, Revised and Expanded, cap.22, C-S. Wu (ed.), New York (2004).

14. Kurata, M. \& Tsunashima, Y. - "Viscosity - Molecular Weight Relationships and Unperturbed Dimensions of Linear Chain Molecules", in: Polymer Handbook, J. Brandup, E. H. Immergut, E. A. Grulke (eds.), John Wiley \& Sons, New York (2003).

15. Ouano, A. C. - J. Polym. Sci. Part A-1: Polym. Chem., 10, p.2169 (1972). http://dx.doi.org/10.1002/pol.1972.150100727

16. Menges, M. \& Schmidt-Naake, G. - Angew. Makromol. Chem., 258, p.51 (1998). http://dx.doi.org/10.1002/(SICI)15229505(19980801)258:1\%3C51::AID-APMC51\%3E3.0.CO;2-T

17. Tribe, K.; Saunders, G. \& Meißner, R. - Macromol. Symp., 236, p. 228 (2006). http://dx.doi.org/10.1002/masy.200690059

18. Hong, L. Z. \& Liu, G. J. - Macromolecules, 43, p.3941 (2010). http:// dx.doi.org/10.1021/ma100353t

19. Çatiker, E. \& Güner, A. - Polym. Bul., 41, p.223 (1998).

20. Cecopieri-Gómez, M. L. \& Palacios-Alquisira, J. - J. Braz. Chem. Soc., 16, p.426 (2005).

21. Lee, J. S.; Kim, S. C. \& Lee, H. K. - Macromol. Res., 16, p.631 (2008). http://dx.doi.org/10.1007/BF03218572

22. Stockmayer, W. H. \& Fixman, M. - J. Polym. Sci. Part C: Polym. Symp., 1, p.137 (1963).

23. Michielsen, S. - "Specific Refractive Index Increments of Polymers in Dilute Solution", in: Polymer Handbook, J. Brandup, E. H. Immergut, E. A. Grulke (eds.), John Wiley \& Sons, New York (2003).

24. Malawer, E. G. \& Senak, L. - "Introduction to Size Exclusion Chromatography", in: Handbook of Size Exclusion Chromatography and Related Techniques Second Edition, Revised and Expanded, cap.22, C-S. Wu (ed.), Marcel Dekker Inc., New York (2004).

25. Sun, S. F. - "Physical chemistry of macromolecules : basic principles and issues", John Wiley \& Sons, Hoboken (2004). http://dx.doi. org/10.1002/0471623571

26. Florea, M. - J. Chromatogr. A, 878, p.1 (2000). http://dx.doi. org/10.1016/S0021-9673(00)00265-X

27. Teraoka, I. - "Polymer Solution An Introdution to Physical Properties", John Wiley \& Sons, Inc., New York (2002).

28. Delpech, M. C.; Coutinho, F. M. B.; Sousa, K. G. M. \& Cruz, R. C. - Polímeros, 17, p.294 (2007). http://dx.doi.org/10.1590/S010414282007000400008

29. Wall, F. T. \& Hiller, L. A. - Annu. Rev. Phys. Chem., 5, p.267 (1954). http://dx.doi.org/10.1146/annurev.pc.05.100154.001411

30. Flory, P. J. - "Principles of Polymer Chemistry", Cornell University Press, Ithaca (1953).
31. Wypych, G. - "Handbook of Solvents", Chem Tec Publishing, Toronto (2001).

32. Pyun, J. \& Matyjaszewski, K. - Macromolecules, 33, p.217 (2000). http://dx.doi.org/10.1021/ma9910787

33. Hirai, T.; Leolukman, M.; Jin, S.; Goseki, R.; Ishida, Y.; Kakimoto, M.-a.; Hayakawa, T.; Ree, M. \& Gopalan, P. - Macromolecules, 42, p.8835 (2009). http://dx.doi.org/10.1021/ma9018944

34. Bevington, J. C. \& Huckerby, T. N. - Eur. Polym. J., 42, p.1433 (2006). http://dx.doi.org/10.1016/j.eurpolymj.2005.12.011

35. Zhang, H-X.; Lee, H-Y.; Shin, Y-J.; Yoon, K-B.; Noh, S-K. \& Lee, D-H. - Polym. Int., 57, p.1351 (2008). http://dx.doi.org/10.1002/pi.2480

36. Moad, G. - Prog. Polym. Sci., 24, p.81 (1999). http://dx.doi. org/10.1016/S0079-6700(98)00017-3

37. Denisov, E. T.; Denisova, T. G. \& Pokidova, T. S. - "Handbook of free radical initiators", John Wiley \& Sons, Inc., Hoboken (2003). http:// dx.doi.org/10.1002/0471721476

38. Nachtigall, S. M. B.; Stedile, F. C.; Felix, A. H. O. \& Mauler, R. S. - J. Appl. Polym. Sci., 72, p.1313 (1999). http://dx.doi. org/10.1002/(SICI)1097-4628(19990606)72:10\%3C1313::AIDAPP11\%3E3.3.CO;2-4

39. Nachtigall, S. n. M. B.; Felix, A. H. O.; Ojeda, T. \& Mauler, R. S. - Polímeros, 8, p.69 (1998). http://dx.doi.org/10.1590/S010414281998000400010

40. Faravelli, T.; Pinciroli, M.; Pisano, F.; Bozzano, G.; Dente, M. \& Ranzi, E. - J. Anal. Appl. Pyrol., 60, p.103 (2001). http://dx.doi. org/10.1016/S0165-2370(00)00159-5

41. Li, Y.; Xie, X.-M. \& Guo, B.-H. - Polymer, 42, p.3419 (2001). http:// dx.doi.org/10.1016/S0032-3861(00)00767-9

42. Bettini, S. H. P. \& Ruvolo, A. C. - J. Appl. Polym. Sci., 107, p.1430 (2008). http://dx.doi.org/10.1002/app.27201

43. Huber, K.; Bantle, S.; Lutz, P. \& Burchard, W. - Macromolecules, 18, p.1461 (1985). http://dx.doi.org/10.1021/ma00149a017

44. Fujita, H. - Macromolecules, 21, p.179 (1988). http://dx.doi. org/10.1021/ma00179a036

45. Morris, R. L.; Amelar, S. \& Lodge, T. P. - J. Chem. Phys., 89, p.6523 (1988). http://dx.doi.org/10.1063/1.455372

46. Von Meerwall, E. D.; Amelar, S.; Smeltzly, M. A. \& Lodge, T. P. Macromolecules, 22, p.295 (1989). http://dx.doi.org/10.1021/ ma00191a054

47. Levenberg, K. - Quart. Appl. Math. , 2, p.164 (1944).

48. Marquardt, D. W. - J. Soc. Ind. Appl. Math., 11, p.431 (1963). http:// dx.doi.org/10.1137/0111030

49. Wagner, H. L. - J. Phys. Chem. Ref. Data, 14, p.1101 (1985). http:// dx.doi.org/10.1063/1.555740

50. Weber, V.; Duval, M.; Ederlé, Y. \& Mathis, C. - Carbon, 36, p.839 (1998). http://dx.doi.org/10.1016/S0008-6223(98)00015-3

51. Orwoll, R. A. \& Arnold, P. A. - "Polymer - Solvent Interaction Parameter c", in: Physical Properties of Polymers Handbook, J. E. Mark (ed.), Springer, New York (2007).

52. Spyriouni, T.; Tzoumanekas, C.; Theodorou, D.; Muller-Plathe, F. \& Milano, G. - Macromolecules, 40, p.3876 (2007). http://dx.doi. org/10.1021/ma0700983

53. Terao, K.; Hokajo, T.; Nakamura, Y. \& Norisuye, T. - Macromolecules, 32, p.3690 (1999). http://dx.doi.org/10.1021/ma990091o

Enviado: $12 / 02 / 11$

Reenviado: $14 / 04 / 11$

Aceito: $16 / 06 / 11$ 